\title{
Les communautés religieuses et l'enseignement au Nouveau-Brunswick depuis 1872
}

\section{Alexandre-J. Savoie}

Volume 48, 1981

URI : https://id.erudit.org/iderudit/1007101ar

DOI : https://doi.org/10.7202/1007101ar

Aller au sommaire du numéro

Éditeur(s)

Les Éditions Historia Ecclesiæ Catholicæ Canadensis Inc.

ISSN

0318-6172 (imprimé)

1927-7067 (numérique)

Découvrir la revue

Citer cet article

Savoie, A.-J. (1981). Les communautés religieuses et l'enseignement au Nouveau-Brunswick depuis 1872. Sessions d'étude - Société canadienne d'histoire de l'Église catholique, 48, 67-77. https://doi.org/10.7202/1007101ar

Tous droits réservés @ Les Éditions Historia Ecclesiæ Catholicæ Canadensis Inc., 1981
Ce document est protégé par la loi sur le droit d'auteur. L'utilisation des services d'Érudit (y compris la reproduction) est assujettie à sa politique d'utilisation que vous pouvez consulter en ligne.

https://apropos.erudit.org/fr/usagers/politique-dutilisation/ 


\section{Les communautés religieuses et l'enseignement au Nouveau-Brunswick depuis 1872}

Avant de vous entretenir du rôle des communautés religieuses dans l'enseignement au Nouveau-Brunswick depuis 1867, il est à propos de rappeler sommairement la situation scolaire avant 1872 '. À la promulgation de la Confédération canadienne, les francophones du NouveauBrunswick jouissaient d'avantages scolaires à peu près comparables à ceux des anglophones : leurs programmes d'études n'étaient pas tellement élaborés, mais il en était de même des programmes anglais. Le gouvernement de la province reconnaissait pratiquement, mais non légalement, les écoles catholiques et les subventionnait comme telles. Quelques-unes figuraient au budget provincial sous l'étiquette "écoles catholiques ». Il en était de même des écoles des autres dénominations religieuses. C'était le système des écoles séparées.

Il est également vrai que les autorités religieuses, tant catholiques que protestantes, ont profité des avantages des lois scolaires de 1847 et de 1858 pour construire des écoles et des académies dans lesquelles on pouvait enseigner librement la religion et déterminer le choix des manuels scolaires. En 1871, il y avait 96010 catholiques au NouveauBrunswick, un peu plus du tiers de la population qui comptait 285594 âmes. Officieusement, il y avait des écoles catholiques dont les écoles françaises faisaient partie. Les Acadiens étaient alors au nombre de 44907 et formaient $15.7 \%$ de la population. Ils vivaient en marge de la société et dans l'oubli presque total des autres Canadiens. Quant à l'enseignement du français dans les écoles, le texte suivant, tiré du Rapport de la Commission de planification académique de l'Université de Moncton, laisse entrevoir l'importance qu'on lui accordait.

À la veille de la Confédération canadienne, le gouvernement du Nouveau-Brunswick, de par les dispositions de ses lois scolaires,

1 On trouvera plus de détails sur les différents aspects de cet exposé dans Alexandre-J. Savole, Un siecle de revendications scolaires au Nouveau-Brunswick (1871-1971), t. 1 : « Du français au compte-gouttes (1871-1936) », Edmundston, 1978, 255 p. 
permettait aux Acadiens de s'instruire sans bruit dans leur langue maternelle. dans une atmosphère conforme à leur culture et à leur loi 2.

\section{LA LOI DES ÉCOLES COMMUNES DE 1871}

Fort de l'article 93 de l'Acte de l'Amérique du Nord Britannique, le ministre George Edwin King entreprit, au nom du gouvernement dı Nouveaul-Rnınswick, de fạire vạloir les droits de lạ province en matière d'instruction. Le 20 avril 1869. il présentait un projet de loi des écoles communes et obtenait de l'Assemblée législative l'autorisation de faire imprimer 500 exemplaires du projet soumis. Le 24 février 1870 , le ministre King revenait à la charge, mais s'apercevant que son projet était voué à l'échec, il le retira de la Chambre. Le gouverneur du temps, Lemuel A. Wilmot, convoqua des élections provinciales pour la fin de juin 1870 . Il avait eu bien soin de changer le chef de l'administration, Andrew Rainsford Wetmore, par G. Edwin King et de s'assurer du concours de George L. Hatheway et Benjamin R. Stevenson.

Le 17 mai 1871, le gouvernement du Nouveau-Brunswick approuvait de justesse la Loi des écoles communes par laquelle la province s'engageait à dispenser gratuitement l'enseignement à tous les enfants, indépendamment de leur sexe, de leur religion et de leur couleur. Cette loi devait corriger la situation déplorable des écoles qui existaient alors. Toutefois, à la dernière minute, les législateurs avaient introduit un article qui devait provoquer l'ire des catholiques. C'était l'article 60 qui excluait l'enseignement de la religion dans les écoles, ainsi que tout emblème se rapportant à une religion. Une lacune : la loi scolaire ne faisait aucune allusion au statut de la langue française. Elle ignorait totalement le fait français, à peine quatre ans après la sanction de la Constitution canadienne qui prônait le respect de la langue des deux peuples fondateurs : l'anglais et le français.

D'après les évêques catholiques du temps, la question confessionnelle était de première importance. Aussi protestèrent-ils énergiquement contre l'application de la loi scolaire de 1871 auprès des gouvernements provincial et fédéral. Ils allèrent jusqu'au Conseil Privé de Londres sans obtenir justice. Leurs démarches ne furent toutefois pas vaines. Le 13 décembre 1873, un premier compromis permettait aux religieuses et religieux de porter l'habit de leur congrégation dans les écoles publiques et d'y enseigner le cathéchisme avant ou après les heures régulières de classe. Le 6 août 1875, un second compromis amendait quelques

2 Rapport paru le 15 décembre 1971, p. 11. 
règlements relatifs à l'école que pouvaient fréquenter les élèves et aux manuels scolaires prescrits par le Bureau d'instruction publique de la province.

\section{AUTRES FORMES DE RÉACTION}

Il y eut d'autres formes de réaction beaucoup plus constructives dont les plus importantes furent la fondation de couvents et de collèges, la fondation de la Société nationale l'Assomption (1851) et de la Société mutuelle l'Assomption (1903), l'organisation de congrès pédagogiques français (1901) et de La Petite Boutique (1922) à Edmundston, l'infiltration au Nouveau-Brunswick de l'Ordre de JacquesCartier (1903) dont les membres ont contribué à fonder plusieurs organismes destinés à corriger la situation éducative, sociale, religieuse et économique des francophones de la province. Il ne sera question dans le présent travail que des couvents et des collèges.

\section{La fondation de courents}

Une des premières réactions à la Loi des écoles communes de 1871 a été la fondation de couvents avec l'encouragement et la bénédiction des évêques pour sauvegarder la foi catholique. Il est relativement facile de démontrer que les religieuses francophones des couvents, fondés entre 1872 et 1880 , ont aussi sauvé l'enseignement du français dans la province du Nouveau-Brunswick. Sans leur dévouement à la cause française, surtout pendant la période de 1872 à 1907, et sans leur contribution personnelle à l'oeuvre éducatrice de l'enseignement, fussent-elles soeurs de la Charité, de la Congrégation de NotreDame ou religieuses Hospitalières de Saint-Joseph, il est certain que le français aurait périclité. L'école publique n'aurait jamais pu préparer des élèves francophones à suivre des cours au département français de l'École Normale de Fredericton car, de 1875 à 1907, il ne s'enseignait pas suffisamment de français dans les écoles publiques pour les y préparer. Les étudiants francophones qui suivront des cours à l'École Normale seront des élèves des couvents et des collèges.

Un des premiers à agir fut le Père Camille Lefebvre, c.s.c., fondateur et supérieur du collège Saint-Joseph de Memramcook. Le Père Lefebvre s'opposa à la nouvelle loi scolaire. Il le fit toutefois sans éclat, sans ostentation et surtout sans haine de personne. Il refusa d'abord la visite annuelle de l'inspecteur des écoles du gouvernement au collège et perdit conséquemment la subvention annuelle de $\$ 800$, somme très importante au bon fonctionnement d'une institution déjà démunie de ressources financières. Le 15 octobre 1873, le Père Lefebvre inaugurait le couvent de Notre-Dame du Sacré-Coeur à Memramcook. Il avait dû surmonter de nombreuses difficultés avant de réussir cet exploit. 
Si la vallée de Memramcook et des environs est demeurée une des parties catholique et française de la province, le crédit en revient en grande partie aux soeurs de la Charité et aux Pères de la Congrégation Sainte-Croix. Ce que les soeurs de la Charité ont fait au couvent de Memramcook, elles l'ont répété au couvent de l'ImmaculéeConception de Bouctouche.

Deux autres congrégations religieuses devaient jouer un rôle de première importance auprès de la population : les religieuses Hospitalières de Saint-Joseph et les soeurs de la Congrégation de Notre-Dame. Les religieuses Hospitalières étaient déjà à Tracadie, comté de Gloucester, depuis le 29 septembre 1868 , où elles se dévouaient au soulagement physique et moral des lépreux. Il ne faut pas s'étonner de les voir ouvrir une école en 1873, grâce à la générosité de quelques paroissiens. Au début. l'oeuvre obtint quelque succès. mais après douze années d'enseignement, les Hospitalières durent revenir à leur oeuvre de prédilection : le soin des lépreux. Toutefois, en 1912, les religieuses Hospitalières ouvraient les portes d'un nouveau couvent à un grand nombre d'élèves pensionnaires et externes. Ainsi naissait l'académie de la Sainte-Famille de Tracadie, appelée à faire un magnifique travail dans la région.

L'oeuvre par excellence des religieuses Hospitalières dans le domaine éducatif est celle de Saint-Basile, comté de Madawaska. Elle a contribué plus que tout autre organisme à sauvegarder le patrimoine des francophones du nord-ouest de la province, sans pour autant nuire aux droits des anglophones. La contribution des religieuses Hospitalières au développement culturel et moral de cette partie du NouveauBrunswick est incommensurable.

Les soeurs de la Congrégation de Notre-Dame ont aussi contribué à relever le niveau d'instruction et à inculquer la fierté nationale à de nombreux Acadiens. Et cela, dès le 6 septembre 1872 à Bathurst, le 16 août 1874 à Caraquet et le 18 août 1874 à Saint-Louis-de-Kent. Il est un fait indéniable, le village de Bathurst-Ouest est la seule partie de la ville de Bathurst qui ait conservé un peu de fierté de parler français. L'Académie de la Sainte-Famille y était pour quelque chose avant l'arrivée du Collège du Sacré-Coeur en 1916. D'ailleurs, cette influence se manifeste aussi dans les deux autres régions où les soeurs de la Congrégation de Notre-Dame ont oeuvré depuis 1874 : Saint-Louis-deKent et Caraquet. Ces deux régions sont demeurées des foyers de culture française au Nouveau-Brunswick. S'il en est ainsi, c'est aussi et surtout grâce au zèle et au dévouement de quelques prêtres. 
De ce qui précède, il est évident que les soeurs de la Charité, de la Congrégation de Notre-Dame et les religieuses Hospitalières de SaintJoseph ont joué un rôle prépondérant, de 1872 à 1907, non seulement pour la sauvegarde de la religion et du français dans la province aux niveaux primaires et intermédiaires, mais aussi pour le développement de la culture française en intéressant les élèves à la musique sous toutes ses formes, à l'art dramatique et aux sciences culinaires. Il suffit de connaître un tant soit peu les régions où leur zèle et leur dévouement se sont exercés pendant cette période pour constater un fait : la vitalité française dans la vallée de Memramcook, dans le comté de Kent, dans la péninsule du comté de Gloucester et dans les comtés de Madawaska et Victoria.

Les réalisations de ces congrégations religieuses se continueront et prendront même de l'ampleur pendant la première moitié du $\mathrm{XX}^{\mathrm{e}}$ siècle au fur et à mesure qu'elles se développeront. Les soeurs de la Charité de Saint-John assumeront la direction des couvents de Shédiac en 1888, de Saint-Anselme en 1915 et lorsque la Congrégation de NotreDame du Sacré-Coeur sera reconnue par Rome, le 23 décembre 1923, les soeurs de Notre-Dame-du-Sacré-Coeur ouvriront un couvent à PetitRocher en 1923, à Moncton et à Grand-Sault en 1924.

Un événement survenu en France à la fin du XIX ${ }^{\mathrm{c}}$ siècle, la persécution contre les congrégations religieuses, devait aussi contribuer à la conservation et à la diffusion de l'enseignement du français en Acadie. Plusieurs congrégations de religieuses et de religieux durent quitter la mère patrie pour s'exiler dans des pays plus accueillants. C'est ainsi qu'arrivèrent au Nouveau-Brunswick les congrégations des Filles de Jésus et des Filles de la Sagesse. Aux premières furent offerts des couvents à Dalhousie en 1903 et à Rogersville en 1904 ; aux secondes, une académie à Edmundston en 1905.

En 1918, les religieuses de Jésus-Marie prendront possession du couvent de Lamèque construit par les paroissiens avec la collaboration de leur curé, J.-Alfred Trudel. Ce couvent suivra les programmes d'études de la province et préparera les jeunes filles à l'École Normale et les garçons à l'enseignement des collèges.

En 1923, $\mathrm{M}^{\mathrm{gr}}$ Arthur Melanson, alors curé de Campbellton, fondait avec l'approbation de son évêque, $\mathrm{M}^{\mathrm{g}}{ }^{\mathrm{r}}$ Patrice-Alexandre Chiasson, c.j.m., une nouvelle congrégation religieuse : les Filles de Marie-del'Assomption. De ces religieuses, Omer Le Gresley, c.j.m., écrira : 
Cette nouvelle société répondait à un besoin réel, car, jusqu'alors, il n'y avait pas de noviciat français de Soeurs enseignantes en Acadie, et nos jeunes Acadiennes désireuses de se dévouer à l'éducation. n'avaient le choix qu' entre un noviciat anglais du pays et un noviciat français d'une autre province, sans savoir si on leur donnerait ensuite la consolation de travailler pour le bien de leurs compatriotes.

Il faudrait aussi souligner le rôle très important joué par les Ursulines dans les couvents de Jacquet River et de Saint-Léonard ; les soeurs Maristes dans les différentes écoles du comté de Madawaska ; les Frères du Sacré-Coeur et les Frères de l'Instruction Chrétienne dans les écoles de la province ; les Pères Salésiens à Jacquet River et Saint-Louis-de-Kent.

En ce qui concerne le rôle joué par les couvents francophones de 1872 à 1937 au Nouveau-Brunswick, les faits démontrent qu'on y a mieux enseigné les deux langues officielles du pays et obtenu de meilleurs résultats que dans les écoles publiques. Dans ces dernières, l'élève anglophone, à quelques exceptions près, est demeuré unilingue anglais, tandis que l'élève francophone fréquentant l'école dans les milieux mixtes n'a appris ni le français ni l'anglais. Il a appris ce qu'un éducateur religieux a appelé un français-bâtard : un français composé d'anglicismes et de barbarismes. Le bilinguisme enseigné dans les écoles publiques depuis 1872 était un bilinguisme à sens unique. Il a été désastreux pour les Acadiens, en assimilant un très grand nombre et encourageant leur mortalité scolaire à un très bas âge.

\section{La fondation de collèges}

Que dire maintenant du rôle joué par nos collèges ${ }^{3}$ ? Selon le sénateur Pascal Poirier, en 1867 on n'aurait pas trouvé un seul avocat, un seul médecin parmi les Acadiens du Nouveau-Brunswick. Il y avait, tout au plus quelques marchands et sept prêtres francophones dans les diocèses de Saint John et Chatham.

\section{LE COLLÈGE SAINT-JOSEPH DE MEMRAMCOOK (1864)}

Fondé en 1864 par le Père Camille Lefebvre, le Collège Saint-Joseph reçut en 1868 pleins pouvoirs de conférer des grades. En 1898, il obtint du gouvernement du Nouveau-Brunswick un amendement à sa charte qui en faisait l'Université du Collège Saint-Joseph. Le Collège s'est borné au cours classique traditionnel jusqu'en 1942, alors qu'il

3 Sur le développement des collèges, voir le Rapport de la Commission de planification académique de l'Université de Moncton, 1971, p. 18-23. 
établissait un autre programme conduisant au baccalauréat en commerce. Dans les deux ans qui suivirent on créait un baccalauréat en sciences et un baccalauréat en pédagogie.

En 1948 commençait le déménagement à Moncton, par étapes, de sorte qu'en 1961 presque tout l'enseignement postsecondaire se donnait dans un immeuble de la rue Church. Trois cents élèves y étaient inscrits à plein temps en arts, en sciences, en pédagogie, en génie et en commerce, tandis qu'à Memramcook quatre-cent cinquante élèves suivaient les deux premières années du cours des arts, le cours secondaire, et des cours d'agriculture organisés en collaboration avec le Gouvernement provincial. En même temps se développaient à un rythme accéléré des cours hors cadre, sous l'autorité d'un directeur permanent : cours du soir conduisant à des grades universitaires ou à des certificats d'études, institut de formation de chefs, et diverses activités organisées avec le concours d'organismes extérieurs. Des cours d'été y ont été organisés à partir de 1938 ; ils groupèrent plus tard jusqu'à quatre cents élèves. Comme on le voit, déjà dans les années '60, Saint-Joseph en était rendu pour ainsi dire à mi-chemin entre le collège classique et l'université proprement dite, avec ses embryons de facultés, son service d'Extension, ses trois collèges affiliés groupant quelque cinq cents élèves au total.

\section{LE COLLÈGE SAINT-LOUIS DE KENT (1874-1882)}

Prétextant que le Collège Saint-Joseph était plus anglais que français, le Père François-Marcel Richard décida, avec l'approbation de l'Ordinaire, de construire un couvent et un collège classique pour les Acadiennes et les Acadiens à Saint-Louis dans le comté de Kent. Le 30 juillet 1874, il annonçait l'ouverture du Collège pour le mois de septembre. Il avait retenu les services de trois professeurs : l'un pour le français, l'autre pour l'anglais et le troisième pour les sciences. Malheureusement, "fortement réprimandé " par $\mathbf{M}^{\mathrm{gr}} \mathrm{James}$ Rogers, évêque du diocèse de Chatham, le Père Richard devait fermer les portes du Collège après huit années d'existence.

\section{LE COLLÈGE DU SACRÉ-COEUR DE CARAQUET (1899) - DE BATHURST (1916)}

Si la loi scolaire de 1871 avait eu de mauvais effets sur le peuple acadien, elle avait aussi eu pour effet d'attirer l'attention de plusieurs Canadiens français influents qui s'étaient faits les défenseurs de la cause scolaire acadienne à Ottawa et à Québec. Un des résultats de ces rencontres avait été la fondation de la Société nationale des Acadiens le 20 
juillet $1881^{4}$. Dans l'espace de quelques années, on vit surgir dans les provinces Maritimes des collèges et des couvents. Le problème de l'enseignement prenait une tournure beaucoup plus intéressante pour les Acadiens.

En 1898, les Pères Eudistes, qui avaient fondé en 1890 le Collège Sainte-Anne à Church Point, en Nouvelle-Écosse, acceptèrent de fonder un collège classique à Caraquet, au nord-est de la province. Le 8 janvier 1899, s'ouvrait le Collège du Sacré-Coeur de Caraquet dans un bâtiment que leur offrait le curé de l'endroit, le futur $\mathrm{M}^{\mathrm{g} r}$ Théophile Allard. Le cours classique était le même que celui de Memramcook. Dès 1900, le Collège prenait le rang d'université avec tous les pouvoirs et privilèges attachés à ce titre, y compris celui de conférer des grades. En 1907, une aile s'ajoutait au collège.

Le 31 décembre 1915, un incendie détruisait le collège de fond en comble. Les Pères Eudistes décidèrent alors de transférer le collège à Bathurst, site plus central et plus commode, surtout à l'époque, au point de vue communications. D'ailleurs ils n'avaient qu'à utiliser un vaste immeuble tout neuf destiné à abriter leur scolasticat. Or, quatorze mois plus tard, le 9 mars 1917, ce collège était à son tour la proie des flammes. C'était le troisième sinistre majeur que subissaient les Eudistes en Acadie, leur collège de Church Point ayant brûlé en 1898. Le Collège fut reconstruit à l'épreuve du feu sur les ruines de l'ancien, grâce à l'immense sympathie qu'avaient suscitée tant de malheurs. La première rentrée en 1921 réunissait cent cinquante élèves. Il y en avait deux cent, quatre ans plus tard. Des cours d'été furent inaugurés en 1936, destinés surtout au personnel enseignant des écoles. Ces cours très suivis ont fait un bien immense à la région, en suppléant à l'insuffisance des programmes de français donnés à l'École normale de Fredericton.

Comme le Collège Saint-Joseph, le Collège du Sacré-Coeur a toujours maintenu le cours classique tant au niveau secondaire que collégial. Normalement, les finissants francophones de $12^{\mathrm{e}}$ année sortant des écoles publiques auraient dû être prêts à passer au niveau collégial. En fait, ils ne l'étaient pas, faute de connaissance et de pratique suffisantes du français. Le Collège a donc institué une année préparatoire de français et de latin à l'adresse des élèves qui avaient terminé leur $12^{\mathrm{e}}$ année. Cette préparation achevée, les élèves passaient en Belles-Lettres, première année du cours collégial.

4 "Les Acadiens des Maritimes : études thématiques ", 1980, Moncton, Centre d'études acadiennes, p. 448-449, "L'Enseignement en Acadie de 1604 à 1970 ", par Alexandre-J. Savoie. 
Le Collège du Sacré-Coeur a dû modifier ses programmes en 1961, en établissant des cours à option en sciences, en mathématiques et en sciences sociales. Les cercles d'études pour adultes du diocèse ont été une des spécialités du Collège depuis 1953. De 1953 à 1961, en moyenne 250 personnes ont assisté chaque année à ces cercles. En 1961, le Collège dut affronter le même problème que celui de Saint-Joseph : le manque d'argent. La présence de nombreux Pères dans l'enseignement avait empêché les déficits de prendre des proportions alarmantes. Comme à Saint-Joseph, les Pères se faisaient rares, et les laïcs qui les remplaçaient commandaient des salaires plus élevés. Que faire dans une telle situation ? Céder les collèges et leurs dépendances à des corporations laïques semblait être la meilleure solution.

\section{LE COLLÈGE SAINT-LOUIS D'EDMUNDSTON (1946)}

Le comté de Madawaska, bassin le plus prolifique en recrues pour les collèges classiques, n'avait jamais pu réussir à se donner un collège à lui, malgré des tentatives répétées pendant un siècle. Il aura fallu la création du diocèse d'Edmundston en 1944 pour décider définitivement de la création d'un collège classique dans la région. Le ler septembre 1946, Saint-Louis d'Edmundston ouvrait ses portes sous la direction des Pères Eudistes. L'année suivante, en vertu de la charte du 3 avril 1947, le Collège était déclaré Université avec pouvoir de conférer des grades. L'édifice principal fut inauguré le 22 octobre 1950.

Le Collège Saint-Louis s'est développé rapidement, au point d'égaler le Collège de Bathurst en peu de temps. Il y avait dans toutes les classes du cours classique quatre cents élèves en 1960. Il a commencé, comme les autres collèges, par le cours classique et à l'instar des autres collèges, ses activités scolaires se sont diversifiées. Une école de musique fut fondée en 1952. Des cours hors cadre furent organisés en coopération, en sociologie, en économie, en animation sociale. En 1960, furent inaugurés des cours d'été à l'adresse des enseignantes et des enseignants.

Contrairement au Collège de Bathurst où le milieu est moins français, le Collège Saint-Louis a vu son cours secondaire très bien fréquenté dès le début, également partagé entre externes et pensionnaires. Le Collège s'est toujours tenu près de la population qui, d'ailleurs, l'a épaulé en toutes circonstances et lui a fourni une aide appréciable. 


\section{LES COLLÈGES AFFILIÉS}

Depuis une trentaine d'années les Collèges Saint-Joseph, du SacréCoeur et Saint-Louis se sont affiliés des collèges, surtout féminins. Les institutions mères conféraient des grades aux élèves qui suivaient certains cours de ces collèges, selon les modalités qui variaient avec chaque institution.

Les collèges affiliés au Collège Saint Joseph n'existent plus. Nous ne faisons que les signaler en passant. Le Collège de l'Assomption, institution pour garçons fondée par le diocèse de Moncton en 1934, donnait en 1960 les deux premières années du cours collégial à quarante-quatre élèves. Le Collège Notre-Dame d'Acadie, à Moncton, institution pour filles dirigée par les soeurs de Notre-Dame du SacréCoeur et affiliée en 1943, offrait les programmes du baccalauréat ès arts et du baccalauréat en musique. En 1961, il y avait soixanteseize élèves au niveau collégial. Le Séminaire Notre-Dame du BonSecours, fondé en 1956 à Humphrey, était destiné au recrutement des Pères Rédemptoristes. En 1961, vingt élèves suivaient le cours collégial.

Les collèges affiliés aux collèges du Sacré-Coeur et Saint-Louis sont aussi disparus. Le Collège Jésus-Marie de Shippagan, dirigé par les religieuses de Jésus-Marie et affilié au Collège de Bathurst en 1960, est devenu le Centre universitaire de Shippagan avec une nouvelle direction. Le Collège Maillet de Saint-Basile, dirigé par les religieuses Hospitalières et affilié au Collège Saint-Louis en 1949, a cessé d'exister. 


\title{
CONCLUSION
}

Telles sont brièvement les grandes lignes du rôle des communautés religieuses dans l'enseignement au Nouveau-Brunswick de 1867 à 1967. Les constatations suivantes méritent d'être considérées sérieusement :

1) Nos couvents ont cessé d'exister après le concile de Vacitan II (1962-1965) et la mise en vigueur de la Loi du programme d'égalité sociale de 1967, alors que le gouvernement provincial assumait la responsabilité financière de l'instruction. $\mathrm{Y}$ a-t-il une relation entre ces deux événements et la disparition des couvents ? Je me garde de porter un jugement, je ne fais que constater un fait.

2) Les Collèges Saint-Joseph, du Sacré-Coeur, Saint-Louis-Máaillet et leurs collèges affiliés ont accepté de suspendre leur charte universitaire pour fonder l'Université de Moncton avec ses trois Centres universitaires : Moncton, Edmundston et Shippagan.

3) L'Université du Sacré-Coeur de Bathurst et ses dépendances ont été vendues au gouvernement provincial en 1976 pour devenir le Collège de technologie francophone du Nouveau-Brunswick. Grâce aux subventions des gouvernements fédéral et provincial, ce Collège est appelé à jouer un rôle très important.

En tant qu'enseignant de la " vieille école ", je ne puis m'empêcher de féliciter et de remercier bien sincèrement les religieuses des différents couvents qui ont oeuvré pendant un siècle pour la cause catholique et française au Nouveau-Brunswick. Quant aux Pères qui ont oeuvré dans les collèges, je fais mienne la citation du Père Marcel Tremblay c.j.m. : «Avec les Pères de la Congrégation de Sainte-Croix, les Eudistes partagent l'honneur d'avoir joué un rôle incontestable de pionniers de l'éducation catholique et française en Acadie ${ }^{5}$ ".

\author{
Alexandre-J. SAvole \\ Edmundston, N.-B.
}

(Inspecteur des écoles du

Nouveau-Brunswick, 1950-1967)

5 Tremblay, Marcel, eudiste, " 50 ans d'éducation, 1899-1949 ", l'Université du Sacré-Coeur, Caraquet - Bathurst, p. 9. 